\title{
A CHARACTERIZATION OF INNER AUTOMORPHISMS
}

\author{
PAUL E. SCHUPP
}

\begin{abstract}
It turns out that one can characterize inner automorphisms without mentioning either conjugation or specific elements. We prove the following

THEOREM Let $G$ be a group and let $\alpha$ be an automorphism of $G$. The automorphism $\alpha$ is an inner automorphism of $G$ if and only if $\alpha$ has the property that whenever $G$ is embedded in a group $H$, then $\alpha$ extends to some automorphism of $H$.
\end{abstract}

It turns out that in the category of groups one can characterize inner automorphisms without mentioning either conjugation or specific elements. It is obvious that if $G$ is a group with $\alpha$ an inner automorphism of $G$ and $G$ is embedded in a group $H$, then $\alpha$ extends to an automorphism of $H$; viz., conjugation by the correct element. Angus Macintyre asked whether or not this extension property actually characterizes inner automorphisms. In this note we prove that it does.

THEOREM. Let $G$ be a group and let $\alpha$ be an automorphism of $G$. The automorphism $\alpha$ is an inner automorphism of $G$ if and only if $\alpha$ has the property that whenever $G$ is embedded in a group $H$ then $\alpha$ extends to some automorphism of $H$.

ProOF. A subgroup $K$ of a group $H$ is malnormal in $H$ if $h K h^{-1} \cap K=\{1\}$ for all $h \in H \backslash K$. We shall prove that any group $G$ is embeddable as a malnormal subgroup of a complete group $H$.

This establishes the theorem, for suppose that the automorphism $\alpha$ of $G$ extends to an automorphism $\beta$ of $H$ where $G$ and $H$ are as in the previous paragraph. Since $H$ is complete, $\beta$ must be an inner automorphism of $H$, say that $\beta(h)=h_{0} h h_{0}^{-1}$. Since $\beta$ extends $\alpha$, we have $h_{0} G h_{0}^{-1}=G$ but since $G$ is malnormal in $H$, we have $h_{0} \in G$ (except possibly in the uninteresting case $G=\{1\}$ ) and thus $\alpha$ is an inner automorphism of $G$.

The theorem in the case where $G$ is countable is really already proved in Miller and Schupp [1]. Here we follow the same idea of using small cancellation products but arrange things to work when the cardinality of $G$ is arbitrary. We shall use only a few well-known results of small cancellation theory (see [2]). We shall construct a certain small cancellation product of $G$ and sufficiently many finite cyclic groups. We use $\sigma, \tau$, and $\lambda$ to denote ordinal numbers.

Let $\left\{g_{\sigma}: \sigma<\lambda\right\}$ be a well-ordered set of generators for $G$. Let

$$
F=G *\left\langle x ; x^{11}\right\rangle *\left(\underset{\sigma<\lambda}{*}\left\langle b_{\sigma} ; b_{\sigma}^{7}\right\rangle\right)
$$

For $\sigma<\lambda$, let

$$
r_{\sigma}=g_{\sigma}\left(x b_{\sigma}\right) x b_{\sigma}^{2}\left(x b_{\sigma}\right)^{2} x b_{\sigma}^{2} \cdots\left(x b_{\sigma}\right)^{80}
$$

Received by the editors June 13, 1986.

1980 Mathematics Subject Classification (1985 Revision). Primary 20E36.

This research was supported by National Science Foundation Grant MCS-80-03251. 
and let

For $\sigma<\tau<\lambda$ let

$$
s_{\sigma}=x b_{\sigma}^{2}\left(x b_{\sigma}\right)^{81} x b_{\sigma}^{2} \cdots\left(x b_{\sigma}\right)^{160}
$$

$$
t_{\sigma, \tau}=\left(x b_{\sigma}\right) x b_{\tau}\left(x b_{\sigma}\right)^{2} x b_{\tau} \cdots\left(x b_{\sigma}\right)^{80} .
$$

Let $R$ be the symmetrized subset of $F$ generated by $\left\{r_{\sigma}, s_{\sigma}, t_{\sigma, \tau}: \sigma<\tau<\lambda\right\}$. It is clear that $R$ satisfies the small cancellation condition $C^{\prime}(1 / 10)$. Let $N$ be the normal closure of $R$ in $F$ and let $H=F / N$. Then $G$ is embedded in $H$. Now no $r \in R$ has a subword of the form $u f u^{-1}$ with $u \neq 1$ and $f$ in a factor of $F$. If $u$ does not end with a letter from the same factor as $f$, then $u f u^{-1}$ is in free product normal form as written. If $u$ is $R$-reduced, then $u f u^{-1} f^{\prime} \neq 1$ in $H$ for any $f^{\prime}$ in a factor of $F$ since small cancellation theory says that any nontrivial word of $F$ which represents the identity of $G$ must contain more than seven-tenths of an element of $R$. Thus $G$ is malnormal in $H$. This argument also shows that $H$ has trivial center.

We must show that $H$ is complete. Clearly, $H$ is generated by $x$ and the $b_{\sigma}$ in view of the relators $r_{\sigma}$. We next note that none of $x$ or the $b_{\eta}$ is contained in the subgroup of $H$ generated by the other generators and $G$. For suppose that some $b_{\eta} \in \operatorname{Gp}\left\{G, x, b_{\sigma}: \sigma \neq \eta\right\}$. Then an equation $b_{\eta}=w$ holds in $H$ where $w$ does not contain $b_{\eta}$. We may suppose that $w$ is $R$-reduced. Now any element of $R$ contains only one $b_{\sigma}$ generator or many occurrences of both $b_{\sigma}$ and $b_{\eta}$. Thus the equation $w b_{\eta}^{-1}=1$ in $H$ cannot hold.

The Torsion Theorem for small cancellation quotients of free products says that the only elements of finite order in $H$ are conjugates of elements of $G$, conjugates of powers of $x$, and conjugates of powers of the $b_{\sigma}$. Knowing all the elements of finite order is a key point in analyzing automorphisms of $H$. Let $\varphi$ be any automorphism of $H$. We need only prove that, up to an inner automorphism, $\varphi$ fixes $x$ and all the $b_{\sigma}$. Since $\varphi(x)$ has order eleven, $\varphi(x)$ is either a conjugate of an element of $G$ or a conjugate of a power of $x$. Following $\varphi$ by an inner automorphism we may suppose that $\varphi(x)$ is in a factor. First suppose that $\varphi(x)=g \in G$. Now $H=\operatorname{Gp}\left\{\varphi(x), \varphi\left(b_{\sigma}\right): \sigma<\lambda\right\}$ but, by the remark above, $x \notin \operatorname{Gp}\left\{G, b_{\sigma}: \sigma<\lambda\right\}$. Thus for some $\eta$ we must have $\varphi\left(b_{\eta}\right)=u g_{1} u^{-1}$ with $g_{1} \in G$ or $\varphi\left(b_{\eta}\right)=u b_{\gamma}^{k} u^{-1}$ where $u$ is $R$-reduced and contains $x$. Following $\varphi$ by another inner automorphism if necessary, we may assume that $u$ does not begin with an element of $G$. In the case that $\varphi\left(b_{\eta}\right)=u b_{\gamma}^{k} u^{-1}$ we have $\varphi\left(s_{\eta}\right)=g u b_{\gamma}^{2 k} u^{-1} \cdots\left(g u b_{\gamma}^{k} u^{-1}\right)^{160}$ which must be equal to the identity in $H$. But the above expression is in free product normal form as written and cannot contain seven-tenths of an element of $R$. Similarly, the case $\varphi\left(b_{\eta}\right)=u g_{1} u^{-1}$ leads to a contradiction. We conclude that $\varphi(x)=x^{j}$ for some $j$. With this fact a similar argument now establishes that each $\varphi\left(b_{\sigma}\right)=b_{\tau}^{k}$ for some choice of $\tau$ and $k$. But now application of $\varphi$ to the relators $s_{\sigma}$ shows that $j$ and each $k$ must be equal to one. For,

$$
\varepsilon\left(s_{\sigma}\right)=x^{j} b_{\gamma}^{2 k}\left(x^{j} b_{\gamma}^{k}\right)^{80} \cdots\left(x^{j} b_{\gamma}^{k}\right)^{160}
$$

and the only way that the latter expression can contain a large part of a relator is to have $j=k=1$. So we conclude that $\varphi$ fixes $x$ and permutes the $b_{\sigma}$.

We now show that $\varphi$ fixes the $b_{\sigma}$. First of all, we show that if $\sigma<\tau, \varphi\left(b_{\sigma}\right)=b_{\gamma}$, and $\varphi\left(b_{\tau}\right)=b_{\eta}$ then $\gamma<\eta$, that is, $\varphi$ is order-preserving on subscripts. For, applying $\varphi$ to $t_{\sigma, \tau}$ we have

$$
\varphi\left(t_{\sigma, \tau}\right)=x b_{\gamma} x b_{\eta}\left(x b_{\gamma}\right)^{2} \cdots\left(x b_{\gamma}\right)^{80}
$$


which must equal the indentity in $H$. The only element of $R$ which $\varphi\left(t_{\sigma, \tau}\right)$ could contain a large part of is $t_{\gamma, \eta}$ which yields $\gamma<\eta$. But now we can conclude the proof, for, by the remark on the minimality of the generating set $\left\{x, b_{\sigma}: \sigma<\lambda\right\}$, all $b_{\sigma}$ must occur in the range of $\varphi$. If $\varphi$ does not fix every $b_{\sigma}$, let $\delta$ be the least subscript such that $\varphi\left(b_{\delta}\right) \neq b_{\delta}$. Then $\varphi\left(b_{\delta}\right)=b_{\eta}$ with $\eta>\delta$. Since $\varphi$ is orderpreserving on subscripts, we have $b_{\delta} \notin \mathrm{Gp}\left\{x, \varphi\left(b_{\sigma}\right): \sigma<\lambda\right\}$ contradicting the fact that the latter set generates $H$. Thus $H$ is complete.

\section{BIBLIOGRAPHY}

1. C. F. Miller and P. E. Schupp, Embeddings into Hopfian groups, J. Algebra 17 (1971), 171-176.

2. P. E. Schupp, A survey of small cancellation theory, Word Problems and the Burnside Problem (Boone, Cannonito, Lyndon eds.), North-Holland, 1972, pp. 569-589.

DEPARTMENT OF MATHEMATICS, UNIVERSity OF ILlinois, URBANA, ILLINOIS 61801 LITP, UNIVERSITÉ PARIS 7, PARIS, FRANCE 